\title{
A Self-Stabilizing Distributed Approximation Algorithm for the Minimum Connected Dominating Set
}

\author{
${ }^{1}$ Tottori University of Environmental Studies \\ Dept. of Information Systems \\ 1-1-1 Wakabadai-kita, Tottori, Japan \\ s-kamei@kankyo-u.ac.jp
}

Sayaka Kamei ${ }^{1}$ and Hirotsugu Kakugawa ${ }^{2}$

\author{
${ }^{2}$ Osaka University \\ Dept. of Computer Science \\ 1-3 Machikaneyama, Toyonaka, Osaka, Japan \\ kakugawa@ist.osaka-u.ac.jp
}

\begin{abstract}
Self-stabilization is a theoretical framework of nonmasking fault-tolerant distributed algorithms. A selfstabilizing system tolerates any kind and any finite number of transient faults, such as message loss, memory corruption, and topology change. Because such transient faults occur so frequently in mobile ad hoc networks, distributed algorithms on them should tolerate such events. In this paper, we propose a self-stabilizing distributed approximation algorithm for the minimum connected dominating set, which can be used, for example, as a virtual backbone or routing in mobile ad hoc networks. The size of the solution by our algorithm is at most $8\left|D_{\text {opt }}\right|+1$, where $D_{\text {opt }}$ is a minimum connected dominating set. The time complexity is $O\left(n^{2}\right)$ steps.
\end{abstract}

\section{Introduction}

\subsection{Self-stabilization}

A distributed system is a set of processes and a set of communication links between the processes. The distributed systems are subject to fail by their very nature. Therefore, fault-tolerance is a major concern in the study of distributed computing.

Self-stabilization [5] is a theoretical framework of nonmasking fault-tolerant distributed algorithms proposed by Dijkstra in 1974. Self-stabilizing algorithms can start execution from an arbitrary (illegitimate) system configuration, and eventually reach a legitimate configuration. That is to say, they do not need all initializations of states of each process and each link. By this property, they tolerate any kind

1-4244-0910-1/07/\$20.00 (c)2007 IEEE. and any finite number of transient faults, such as message loss, memory corruption, and topology change, and can adjust to dynamic changes of the system [6]. In addition, a self-stabilizing system does not need a global and synchronized initialization of each process at all when a system is started, because we can consider that such a starting configuration is a configuration just after transient faults occur. However, by these properties, design of a self-stabilizing algorithm is difficult.

\subsection{Connected dominating set}

In 1979, Sampathkumar et al. introduced the connected dominating set (CDS) problem in graph theory [13]. Let $G=(V, E)$ be an undirected connected graph, where $V$ is a set of nodes and $E$ is a set of edges. A dominating set of $G$ is a subset $D \subseteq V$ such that each member of $V \backslash D$ is adjacent to at least one member of $D$. A set $D^{\prime} \subseteq V$ is a CDS of $G$ if and only if $D^{\prime}$ is a dominating set and the induced subgraph by $D^{\prime}$ is connected. The minimum CDS problem is finding a CDS of the minimum size.

A CDS is useful in the computation of message routing and other network problems for mobile ad hoc networks. Ad hoc networks are collection of wireless mobile nodes, and have no physical backbone infrastructure and no centralized administration. Therefore, a CDS formed by processes can be used for virtual backbone, which plays an important role for routing and connectivity management etc. However, it is known that this problem is NP-hard [4] in unit disk graph model that is one of models of mobile ad hoc networks. In a unit disk graph, there is a link between two nodes if and only if their geographical distance is at most one unit. In mobile ad hoc networks, message loss and topology change occur frequently. Thus, distributed algorithms for mobile ad hoc networks should tolerate such events. 
We propose a self-stabilizing distributed approximation algorithm to compute a minimum CDS of an underlying network $G$ assuming that a BFS (i.e., breadth-first spanning) tree $T$ of $G$ rooted at a process $P_{r}$ is given. An approximation algorithm for the minimization problem is an algorithm which guarantees the approximation ratio $D_{\text {alg }} / D_{\text {opt }}$, where $D_{a l g}$ is the size of the solution of the approximation algorithm in the worst case and $D_{o p t}$ is the size of the optimal solution.

\subsection{Related works}

Because a CDS can be used for virtual backbone for routing messages in ad hoc networks, many algorithms for the CDS have been proposed. The literature [2] is a good survey for this problem in ad hoc networks.

In [8], Guha et al. propose a centralized approximation algorithm for the minimum CDS in unit disk graphs, and its approximation ratio is $O(H(\Delta))$, where $\Delta$ is the maximum degree and $H()$ is the harmonic function. In [12], Marathe et al. propose a heuristic approximation algorithm for the minimum CDS in unit disk graphs, and the size of the solution by their algorithm is at most $8\left|D_{\text {opt }}\right|+1$. .

In [1], Bharghavan et al. propose a distributed approximation algorithm based on the centralized algorithm in [8]. Its approximation ratio is $O(H(\Delta))$, and both its message complexity and time complexity are $O\left(n^{2}\right)$. In [17], Wu et al. propose a different distributed approximation algorithm in unit disk graphs. Its approximation ratio is $O(n)$, and its message complexity and time complexity are $\Theta(m)$ and $O\left(n^{3}\right)$, respectively, where $m$ is the number of the edges in the unit-disk graph.

There exist some distributed approximation algorithms each of which approximation ratio is a constant. In [3], Cheng et al. propose a distributed approximation algorithm in unit disk graphs. The approximation ratio is 8 , respectively. Message complexity and time complexity of the both algorithms are $O(n)$ and $O(n \Delta)$, respectively. In [16], Wan et al. propose a distributed approximation in unit disk graphs. The approximation ratio is 8 , and message complexity and time complexity are $O(n \log n)$ and $O(n)$, respectively. In[18], Wu et al. propose a distributed approximation with approximation ratio $O(1)$ in unit disk graphs. In [7], Gao et al. also propose a distributed approximation in unit disk graphs. Its approximation ratio is $O(1)$, and both its message complexity and time complexity are $O(n)$. However, it is not easy to convert these algorithms into selfstabilizing distributed algorithms. Unfortunately, none of these algorithms are self-stabilizing.

In [11], Jain et al. propose a self-stabilizing algorithm for finding a CDS in $O\left(n^{2}\right)$ steps. However, their algorithm

Marathe et al. proved the approximation ratio of their algorithm was 10 in [12]. However, we proof that later. is not approximation algorithm, i.e., their algorithm does not guarantee its approximation ratio.

Therefore, our algorithm is the first self-stabilizing distributed approximation algorithm for the minimum CDS. Because our algorithm is based on the algorithm in [12] in unit disk graphs, the size of the solution by proposed algorithm is at most $8\left|D_{\text {opt }}\right|+1$, and the time complexity is $O\left(n^{2}\right)$ steps.

\subsection{Organization of this paper}

This paper is organized as follows. In section 2, we formally describe system model and the self-stabilizing distributed minimum CDS problem. In section 3, we present an outline of a heuristic algorithm of Marathe et al. [12] on which our algorithm is based. In section 4, we propose a self-stabilizing approximation algorithm for the minimum CDS. We assume that a BFS tree is given. In section 5, we show proof of correctness of the proposed algorithm, and show performance analysis. In section 6 , we give conclusion of this paper and discuss future works.

\section{Preliminary}

\subsection{System model}

Let $V=\left(P_{1}, P_{2}, \ldots, P_{n}\right)$ be a set of processes (nodes) and $E \subseteq V \times V$ be a set of bidirectional communication links in a distributed system. The number of processes is denoted by $n$. Then, the topology of the distributed system is represented as an undirected graph $G=(V, E)$. We assume that the graph is connected and simple. In this paper, we use "graphs" and "distributed systems", interchangeably. By $N_{i}$, we denote a set of neighbor processes of $P_{i}$. For each process $P_{i}$, process identifier and a set $N_{i}$ are given as constants. Let the distance between $P_{i}$ and $P_{j}$ be the number of the edges on the shortest path between them.

As communication model, we assume that each process can read local state of neighbor processes without delay. This model is called the state reading model. Each process can update its own local state only, but each process can read local state of neighbor processes.

A set of local variables defines local state of a process. By $Q_{i}$, we denote local state of each process $P_{i} \in V$. A tuple of local state of each process $\left(Q_{1}, Q_{2}, \ldots, Q_{n}\right)$ forms a configuration of a distributed system. Let $\Gamma$ be a set of all configurations.

An algorithm of each process $P_{i}$ is given as a set of guarded commands:

$$
*\left[\operatorname{Grd}_{1} \rightarrow \operatorname{Act}_{1} \square \operatorname{Grd}_{2} \rightarrow \operatorname{Act}_{2} \cdots\right]
$$

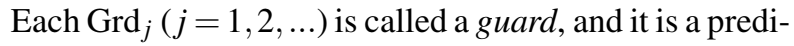
cate on $P_{i}$ 's local state and local states of its neighbors. Each 
Act ${ }_{j}$ is called an action, and it updates local state of $P_{i}$; next local state is computed from current local states of $P_{i}$ and its neighbors. We say that $P_{i}$ is privileged in a configuration $\gamma$ if and only if at least one guard of $P_{i}$ is true in $\gamma$. An atomic step of each process $P_{i}$ consists of the following three substeps: (1) read local states of neighbor processes and evaluate guards, (2) execute a command that is associated to a true guard, and (3) update its local state.

Executions of processes are scheduled by an external (virtual) scheduler. A scheduler decides which process to execute in the next step. At each step, a scheduler selects only one privileged process arbitrarily, and a selected process executes an atomic step. This type of scheduler is known as the central daemon. A scheduler is fair if privileged process is eventually selected to execute by a scheduler, otherwise, it is unfair. We assume the unfair centralized daemon in this paper. Because a privileged process may not be executed forever under an unfair scheduler, an algorithm must be correct with respect to every execution scheduling. Thus, a scheduler is an adversary against an algorithm.

\subsection{Self-stabilization}

For any configuration $\gamma$, let $\gamma^{\prime}$ be any configuration that follows $\gamma$. Then, we denote this transition relation by $\gamma \rightarrow \gamma^{\prime}$. For any configuration $\gamma_{0}$, a computation $E$ starting from $\gamma_{0}$ is a maximal (possibly infinite) sequence of configurations $E=\gamma_{0}, \gamma_{1}, \gamma_{2}, \ldots$ such that $\gamma_{t} \rightarrow \gamma_{t+1}$ for each $t \geq 0$.

Definition 1 (Self-Stabilization) Let $\Gamma$ be a set of all configurations. A system $S$ is self-stabilizing with respect to $\Lambda$ such that $\Lambda \subseteq \Gamma$ if and only if it satisfies the following two conditions:

- Convergence: Starting from an arbitrary configuration, a configuration eventually becomes one in $\Lambda$, and

- Closure: For any configuration $\lambda \in \Lambda$, any configuration $\gamma$ that follows $\lambda$ is also in $\Lambda$ as long as the system does not fail.

Each $\gamma \in \Lambda$ is called a legitimate configuration.

\subsection{Formal definition of the problems}

Definition $2 A$ dominating set of a graph $G=(V, E)$ is a subset $V^{\prime} \subseteq V$ such that there exists $v \in V^{\prime}$ and $(u, v) \in E$ for any $u \in V \backslash V^{\prime}$. A dominating set $V^{\prime}$ of $G$ is minimal if no proper subset of $V^{\prime}$ is a dominating set of $G$. A dominating set $V^{\prime}$ of $G$ is minimum if $\left|V^{\prime}\right| \leq\left|V^{\prime \prime}\right|$ for any dominating set $V^{\prime \prime}$ of $G$.
Definition 3 An independent set of a graph $G=(V, E)$ is a subset $V^{\prime} \subseteq V$ such that $(u, v) \notin E$ for any $u, v \in V^{\prime}$. An independent set $V^{\prime}$ of $G$ is maximal if no proper superset of $V^{\prime}$ is an independent set of $G$.

We call maximal independent sets MISs. It is clear that a subset of $V$ is a minimal dominating set if it is an MIS.

Definition $4 A$ connected dominating set of a graph $G=$ $(V, E)$ is a dominating set $V^{\prime} \subseteq V$ such that an induced subgraph by $V^{\prime}$ is connected. A connected dominating set $V^{\prime}$ of $G$ is minimum if $\left|V^{\prime}\right| \leq\left|V^{\prime \prime}\right|$ for any connected dominating set $V^{\prime \prime}$ of $G$.

We call the members of the CDS dominators, and others dominatees. Each dominatee is dominated by a dominator.

We consider solving the minimum CDS problem in distributed systems in this paper. We assume that each process $P_{i}$ does not know global information of the network, and they know local information $N_{i}$ which is a set of neighbors of $P_{i}$. Under such assumption, we defined the distributed minimum CDS problem as follows.

Definition 5 Let $G=(V, E)$ be a graph that represents a distributed system, let dom $\left(P_{i}\right)$ be a variable that represents whether $P_{i}$ is in a minimum connected dominating set. The distributed minimum connected dominating set problem is a problem defined as follows.

- Each process $P_{i} \in V$ must decide its decision $\operatorname{dom}\left(P_{i}\right) \in$ \{true, false $\}$ as output of $P_{i}$, and

- The set $\left\{P_{i} \in V \mid \operatorname{dom}\left(P_{i}\right)=\right.$ true $\}$ is a minimum connected dominating set of $G$.

We assume that each process $P_{i}$ has a local variable $\operatorname{dom}\left(P_{i}\right)$.

\section{Marathe et al.'s algorithm}

Marathe et al. proposed a sequential heuristic algorithm for the minimum CDS in unit disk graphs [12], and we present outline of their algorithm.

The outline is described more formally in Figure 1. By $G(C)$, we denote an induced subgraph of $G$ by a subset $C$ of $\mathrm{V}$.

Figure 2 illustrates an execution example of Marathe et al.'s algorithm. First, their algorithm selects an arbitrary node $v$ from $G$, and constructs a BFS tree $T$ of $G$ rooted at $v$. Figure 2(a) illustrates a BFS tree. For any node $v_{i}$, let $L\left(v_{i}\right)$ denote the distance from $v$ to $v_{i}$. Let $k$ denote the depth of $T$ (i.e., the maximum distance) in $G$, and let $L_{d}$ be the set of nodes which have the distance $d$ from the root $(0 \leq d \leq k)$. In Figure 2(a)-Figure 2(d), the number in each node $v_{i}$ is the 


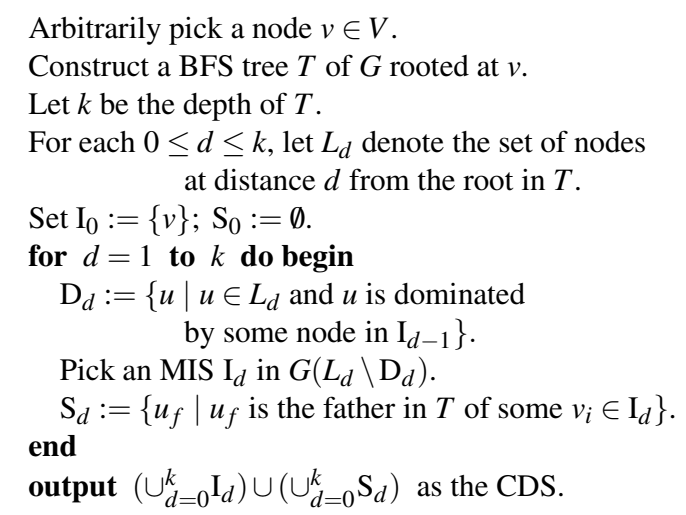

Figure 1. Marathe et al.'s Algorithm

distance $L\left(v_{i}\right)$ from the root on the BFS tree, and each arrow represents a father of the node. Figure 2(b)(resp. 2(c), 2(d)) illustrates the configuration after execution of a for-loop of Figure 1 with $d=1$ (resp. 2, 3), and thick nodes represent that the algorithm has processed the nodes.

The CDS by the heuristic is the union of two subsets of nodes, i.e., $\left(\cup_{d=0}^{k} I_{d}\right) \cup\left(\cup_{d=0}^{k} S_{d}\right)$.

- The first subset $\cup_{d=0}^{k} I_{d}$ is an MIS for $G$. The root $v$ definitely joins a set $I_{0}$. Let $D_{d}$ be a set of nodes $v_{i} \in$ $L_{d}$ each of which is dominated by some node in $I_{d-1}$. For each $1 \leq d \leq k$, a set $I_{d}$ is an MIS in an induced subgraph by $L_{d} \backslash D_{d}$. That is to say, the heuristic paves the field dominated by the members of $I$ in order of increasing of $L\left(v_{i}\right)$ from $v$. (In Figure 2(a)-Figure 2(d), black nodes are in $I_{0}$ or $I_{2}$.)

- The second subset is $\cup_{d=0}^{k} S_{d}$, where a set $S_{d}$ of nodes which are fathers of the members of $I_{d}$ for each $1 \leq d \leq$ $k$. Note that $S_{d} \subseteq L_{d-1}$. (In Figure 2(c)-Figure 2(d), gray nodes are in $S_{2}$.)

We call the above way of construction of an MIS $\cup_{d=0}^{k} I_{d}$ "paving on a BFS tree". For any set $C \subset V$ and any node $v_{i} \notin C$, let the distance between $v_{i}$ and $C$ be the minimum distance between $v_{i}$ and any $v_{j} \in C$. On the MIS constructed by paving on a BFS tree, the set satisfies the following property.

Theorem 1 Let I' be the MIS constructed by paving on a BFS tree T. For any $v_{i}$ in $I^{\prime}$, the distance between $v_{i}$ and $I^{\prime} \backslash\left\{v_{i}\right\}$ is exactly two hops.

The proof of this theorem is the same as one in [16]. Because of limitation of space, proof of this theorem is omitted.

By Theorem 1, the connectivity of the CDS is ensured. By the proof of Theorem 1, in the MIS constructed by paving on $T$, each member $v_{i} \in L_{d}$ of the MIS has a father on $T$ which is neighbor to at least one member of MIS in $L_{d-1}$ or $L_{d-2}$. Therefore, the union of the MIS and a set of fathers of the members of the MIS are connected. Because the MIS is also a minimal dominating set, the union is a CDS. It is clear that, if each member $v_{i} \in L_{d}$ of any MIS has a father which is neighbor to at least one member of the MIS in $L_{d-1}$ or $L_{d-2}$ on $T$, then it can be said that the MIS is constructed by paving on $T$. The CDS is illustrated in Figure 2(e).

We define such a CDS as $C D S$-tree formally as follows:

Definition 6 Let $I^{\prime}$ be any MIS constructed by paving on a BFS tree $T$ for $G$. Let $S^{\prime}(\neq \emptyset)$ be a set of nodes each of which is the father of a member in $I^{\prime}$ on $T$. A set of nodes $I^{\prime} \cup S^{\prime}$ is a CDS-tree of $T$.

Theorem 2 Let $D_{\text {opt }}$ is the minimum CDS. Any CDS-tree is an approximation for the minimum CDS which size is at most $8\left|D_{\text {opt }}\right|+1$ in unit disk graphs.

The proof of the approximation ratio is similar to the one for Wan et al.'s algorithm in [16]. We give the proof follow.

Proof: We consider a CDS-tree $I^{\prime} \cup S^{\prime}$, where $I^{\prime}$ be a maximal independent set constructed by paving on a BFS tree and $S^{\prime}$ be a set of nodes each of which is the father of a member in $I^{\prime}$ on a BFS tree. Let $D_{o p t}$ be any minimum CDS.

First, we consider the size of $I^{\prime}$. Let $v_{1}$ be an arbitrary node in $D_{\text {opt }}$. Let $I_{1}^{\prime}$ be the subset of $I^{\prime}$ such that each member of it is neighbor to $v_{1}$. Then, the size of $I_{1}^{\prime}$ is at most 5 . After we count $I_{1}^{\prime}, I_{2}^{\prime}, \cdots, I_{i-1}^{\prime}$, for $2 \leq i \leq\left|D_{\text {opt }}\right|$, let $v_{i} \in D_{\text {opt }}$ be an arbitrary neighbor of $v_{1}, v_{2}, \cdots, v_{i-2}$ or $v_{i-1}$. Let $I_{i}^{\prime}$ be a subset of $I^{\prime} \backslash\left\{I_{1}^{\prime} \cup I_{2}^{\prime} \cup \cdots I_{i-1}^{\prime}\right\}$ such that each member of it is neighbor to $v_{i}$. Because $v_{i}$ is neighbor to a node $v^{\prime}$ where $v^{\prime}$ is $v_{1}, v_{2}, \cdots, v_{i-2}$ or $v_{i-1}$, the members of $I_{i}^{\prime}$ are in a part of a unit cycle around $v_{i}$ such that it does not overlap with a unit cycle around $v^{\prime}$. That is, the members of $I_{i}^{\prime}$ are only in a field which is smaller than a 240 degree sectoral field around $v_{i}$. (See figure 3.) Therefore, the size of $I_{i}^{\prime}$ is at most 4 for $2 \leq i \leq\left|D_{\text {opt }}\right|$, let $v_{i} \in D_{\text {opt }}$. Therefore, the size of $I^{\prime}$ is

$$
\begin{aligned}
\left|I^{\prime}\right| & =\sum_{i=1}^{\left|D_{\text {opt }}\right|}\left|I_{i}^{\prime}\right|=\left|I_{1}^{\prime}\right|+\sum_{i=2}^{\left|D_{\text {opt }}\right|}\left|I_{i}^{\prime}\right| \\
& \leq 5+4\left(\left|D_{\text {opt }}\right|-1\right) \\
& =4\left|D_{\text {opt }}\right|+1 .
\end{aligned}
$$

Next, we consider the size of $S^{\prime}$. Because each member of $I^{\prime}$ which is not the root of the BFS tree has a father in $S^{\prime}$, the size of $S^{\prime}$ is at most $4\left|D_{\text {opt }}\right|$.

Therefore, the size of the CDS-tree is

$$
\left|I^{\prime} \cup S^{\prime}\right|=\left|I^{\prime}\right|+\left|S^{\prime}\right| \leq 8\left|D_{o p t}\right|+1 .
$$




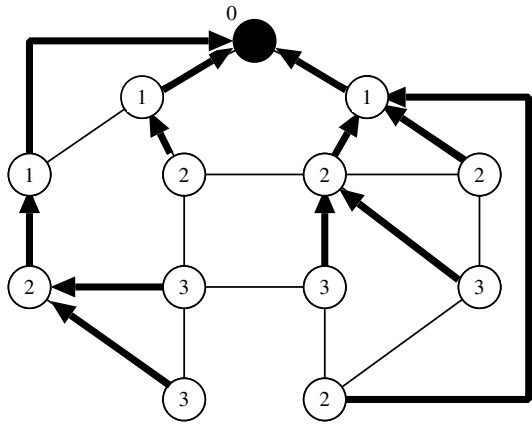

(a) BFS tree

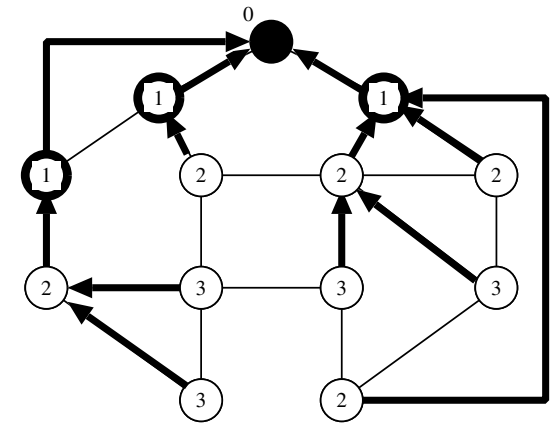

(b) $d=1$

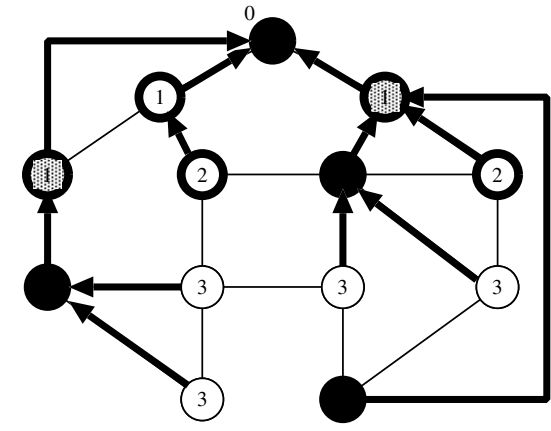

(c) $d=2$

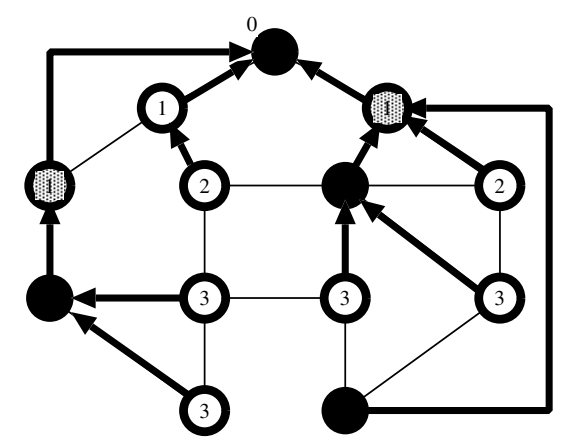

(d) $d=3$

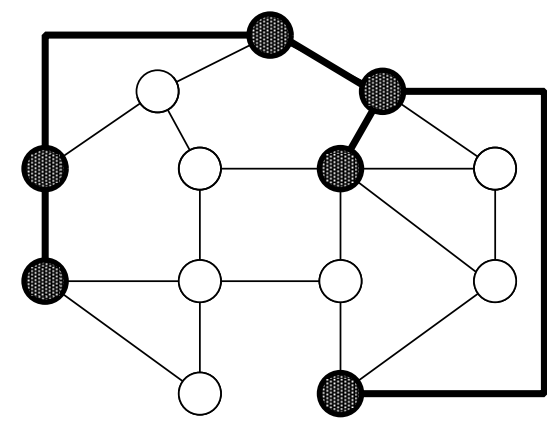

(e) CDS

Figure 2. Outline of Marathe et al.'s algorithm

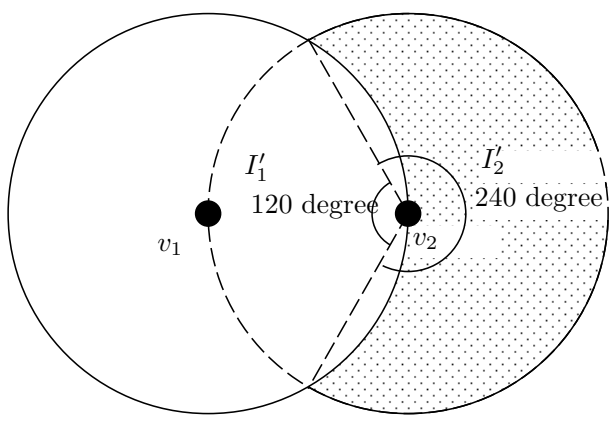

Figure 3. The relationship between $I_{1}^{\prime}$ and $I_{2}^{\prime}$.

\section{Proposed algorithm}

We propose a self-stabilizing algorithm SS-CDS to find a CDS-tree. Our algorithm computes a BFS tree rooted at $P_{r}$ for $G$. Because algorithms for computing a BFS tree have been proposed so far, for example [10,15], we simply adopt one of them. We assume, without loss of generality, that each process $P_{i}$ has the following two variables as output of the BFS tree $T$, and as input of SS-CDS.
- $F\left(P_{i}\right)$ - a process id of the father of $P_{i}$ on $T$.

- $L\left(P_{i}\right)$ - the distance from the root $P_{r}$ to $P_{i}$ on $T$.

The output of each process is

- $\operatorname{dom}\left(P_{i}\right)$ - a boolean if $P_{i}$ is a member of the CDS.

First, SS-CDS computes an MIS. For constructing an MIS, there exist many self-stabilizing algorithms, for example $[14,9]$. However, these algorithms do not ensure that a computed MIS satisfies Theorem 1 on $T$.

Formal description of proposed algorithm SS-CDS is shown in Figure 4. There are five guarded-commands (GC1-GC5) in the algorithm.

- By GC1, the root $P_{r}$ of $T$ with $L\left(P_{r}\right)=0$ joins both the MIS and the CDS.

- By GC2 and GC3, process $P_{i}\left(\neq P_{r}\right)$ decides if $P_{i}$ joins the MIS.

- If each neighbor $P_{j} \in N_{i}$ at which $L\left(P_{i}\right) \geq L\left(P_{j}\right)$ holds is not a member of the MIS, then $P_{i}$ joins the MIS by GC2.

- If there exists a neighbor $P_{j}$ such that $L\left(P_{i}\right) \geq$ $L\left(P_{j}\right)$ holds and $P_{j}$ is a member of the MIS, then $P_{i}$ leaves the MIS by GC3. 
- By GC4 and GC5, process $P_{i}\left(\neq P_{r}\right)$ decides if $P_{i}$ joins the CDS.

- If $P_{i}$ or its at least one child on $T$ is a member of the MIS, then $P_{i}$ joins the CDS by GC4.

- Otherwise, $P_{i}$ leaves the CDS by GC5.

By $\Gamma$, we denote a set of all configurations of SS-CDS. A set of legitimate configurations is defined as follows.

Definition 7 A configuration $\gamma$ is legitimate iff the following three conditions are satisfied.

- Condition 1: $\gamma$ is in $\Gamma_{I}$, where $\Gamma_{I} \subset \Gamma$ is a set of configurations such that a set of processes $\left\{P_{i} \mid\right.$ ind $\left(P_{i}\right)=$ true $\}$ is an MIS of $G$ which satisfies Theorem 1 on $T$,

- Condition 2: ind $\left(P_{i}\right)=$ true implies $\operatorname{dom}\left(P_{i}\right)=$ true for each $P_{i}$, and

- Condition 3: a set $\left\{P_{i} \mid \operatorname{dom}\left(P_{i}\right)=\right.$ true $\}$ is a CDS-tree of $G$.

By $\Lambda_{C}$, we denote a set of legitimate configurations of SSCDS.

\section{Proof of correctness}

Lemma 1 No process is privileged in configuration $\gamma$ if and only if $\gamma \in \Lambda_{C}$.

Proof: Let $\gamma$ be a configuration in which no process is privileged. Assume that $\gamma \notin \Lambda_{C}$.

- Suppose that Condition 1 is false, i.e., $\left\{P_{i} \mid \operatorname{ind}\left(P_{i}\right)=\right.$ true $\}$ is not an MIS which satisfies Theorem 1 on $T$. That is, the set $\left\{P_{i} \mid \operatorname{ind}\left(P_{i}\right)=\right.$ true $\}$ is not an independent set, is not maximal, or does not satisfy Theorem 1 on $T$.

- We assume that the set is not an independent set, i.e., there exists $P_{i}$ and $P_{j}$ in the set such that they are neighbor each other. If $L\left(P_{i}\right)=$ $L\left(P_{j}\right) \neq 0$, the guard of GC3 is true at $P_{i}$ and $P_{j}$ because ind $\left(P_{i}\right)=\operatorname{ind}\left(P_{j}\right)=$ true. Similarly, if $0 \leq L\left(P_{i}\right)<L\left(P_{j}\right)$ (resp. $L\left(P_{i}\right)>L\left(P_{j}\right) \geq 0$ ), then the guard of GC3 is true at $P_{j}$ (resp. $P_{i}$ ). This is a contradiction for the assumption that no process is privileged in $\gamma$. Therefore, the set $\left\{P_{i} \mid \operatorname{ind}\left(P_{i}\right)=\right.$ true $\}$ is an independent set.

- We assume that the set is not maximal. That is, there exists a process $P_{i}$ with $\operatorname{ind}\left(P_{i}\right)=$ false which has no neighbor $P_{j}$ with $\operatorname{ind}\left(P_{j}\right)=$ true. If $L\left(P_{i}\right)=0$, then the guard of $\mathrm{GC} 1$ is true at
Constants — read only and automatically updated $N_{i}$ : The set of neighbors of $P_{i}$ in underlying network $G$. $F\left(P_{i}\right)$ : A father of $P_{i}$ in $T$.

$L\left(P_{i}\right)$ : The distance from the root in $T$.

\section{Variables}

$\operatorname{ind}\left(P_{i}\right)$ : True iff $P_{i}$ is a member of an MIS.

$\operatorname{dom}\left(P_{i}\right)$ : True iff $P_{i}$ is a member of a CDS.

Macro

$\operatorname{Grd}_{k}(k=2,3)$ : The guard of GCk

A Set of Guarded-Commands:

$*[$

/* GC1: Root joins an MIS and a CDS. */

$L\left(P_{i}\right)=0 \wedge\left\{\operatorname{ind}\left(P_{i}\right)=\right.$ false $\vee \operatorname{dom}\left(P_{i}\right)=$ false $\}$ $\rightarrow \operatorname{ind}\left(P_{i}\right):=\operatorname{true} ; \operatorname{dom}\left(P_{i}\right):=$ true;

/* GC2: Join an MIS. */

$L\left(P_{i}\right) \neq 0 \wedge \operatorname{ind}\left(P_{i}\right)=$ false $\wedge$

$\forall P_{j} \in N_{i}\left[L\left(P_{j}\right)>L\left(P_{i}\right) \vee \operatorname{ind}\left(P_{j}\right)=\right.$ false $]$ $\rightarrow \operatorname{ind}\left(P_{i}\right):=$ true;

/* GC3: Leave an MIS. */

$L\left(P_{i}\right) \neq 0 \wedge$ ind $\left(P_{i}\right)=$ true $\wedge$

$\exists P_{j} \in N_{i}\left[L\left(P_{j}\right) \leq L\left(P_{i}\right) \wedge \operatorname{ind}\left(P_{j}\right)=\right.$ true $]$ $\rightarrow \operatorname{ind}\left(P_{i}\right):=$ false;

/* GC4: Join a CDS. */

$L\left(P_{i}\right) \neq 0 \wedge \neg \operatorname{Grd}_{2} \wedge \neg \operatorname{Grd}_{3} \wedge \operatorname{dom}\left(P_{i}\right)=$ false $\wedge$

$\left\{\exists P_{j} \in N_{i}\left[F\left(P_{j}\right)=P_{i} \wedge\right.\right.$ ind $\left(P_{j}\right)=$ true $] \vee$

$\operatorname{ind}\left(P_{i}\right)=$ true $\}$ $\rightarrow \operatorname{dom}\left(P_{i}\right):=$ true;

/* GC5: Leave a CDS. */

$L\left(P_{i}\right) \neq 0 \wedge \neg \operatorname{Grd}_{2} \wedge \neg \operatorname{Grd}_{3} \wedge \operatorname{dom}\left(P_{i}\right)=$ true $\wedge$

$\left\{\forall P_{j} \in N_{i}\left[F\left(P_{j}\right) \neq P_{i} \vee \operatorname{ind}\left(P_{j}\right)=\right.\right.$ false $] \wedge$ $\operatorname{ind}\left(P_{i}\right)=$ false $\}$ $\rightarrow \operatorname{dom}\left(P_{i}\right):=$ false;

Figure 4. SS-CDS: A self-stabilizing approximation algorithm for the minimum CDS

$P_{i}$. If $L\left(P_{i}\right) \neq 0$, then $\forall P_{j} \in N_{i}\left[L\left(P_{j}\right)>L\left(P_{i}\right) \vee\right.$ $\operatorname{ind}\left(P_{j}\right)=$ false $]$ holds at $P_{i}$, and the guard of GC2 is true at $P_{i}$. This is a contradiction for the assumption that no process is privileged in $\gamma$. Therefore, the set $\left\{P_{i} \mid \operatorname{ind}\left(P_{i}\right)=\right.$ true $\}$ is an MIS.

- We assume that the set does not satisfy Theorem 1 on $T$. By proof of Theorem 1, this assumption means that there exists a process $P_{i} \neq P_{r}$ with $\operatorname{ind}\left(P_{i}\right)=$ true whose father $P_{f}(=$ $\left.F\left(P_{i}\right) \in N_{i}\right)$ with $\operatorname{ind}\left(P_{f}\right)=$ false on $T$ is not adjacent to another process $P_{j}$ with ind $\left(P_{j}\right)=$ true and $L\left(P_{j}\right) \leq L\left(P_{f}\right)$. Because $L\left(P_{f}\right)=L\left(P_{i}\right)-1$, this assumption implies that $\forall P_{j} \in N_{f}\left[L\left(P_{j}\right)>\right.$ $L\left(P_{f}\right) \vee \operatorname{ind}\left(P_{j}\right)=$ false]. Then, the guard of GC2 is true at $P_{f}$. This is a contradiction for the assumption that no process is privileged in $\gamma$. 
Therefore, the set $\left\{P_{i} \mid \operatorname{ind}\left(P_{i}\right)=\right.$ true $\}$ satisfies Theorem 1 on $T$.

Therefore, the Condition 1 is true in $\gamma$.

- Suppose that Condition 2 is false, i.e., there exists a process $P_{i}$ with $\operatorname{ind}\left(P_{i}\right)=$ true, but $\operatorname{dom}\left(P_{i}\right)=$ false. If $L\left(P_{i}\right)=0$, then the guard of $\mathrm{GC} 1$ is true at $P_{i}$. If $L\left(P_{i}\right) \neq 0$, then the guard of GC4 is true at $P_{i}$. This is a contradiction for the assumption that no process is privileged in $\gamma$. Therefore, the Condition 2 is true in $\gamma$.

- Suppose that Condition 3 is false. That is, a set $\left\{P_{i} \mid \operatorname{dom}\left(P_{i}\right)=\right.$ true $\}$ is not a CDS-tree of $G$.

- Assume that the set is not a CDS. By the Condition 2, the set is a dominating set because it is clear that an MIS is a dominating set. Therefore, the set is not connected. By Definition 6, there exists a process $P_{i}$ with $\operatorname{dom}\left(P_{i}\right)=$ false which is a father of $P_{j}$ with $\operatorname{ind}\left(P_{j}\right)=$ true for the assumption. Then, the guard of GC4 is true at $P_{i}$. This is a contradiction for the assumption that no process is privileged in $\gamma$. Therefore, the set is a CDS.

- Assume that the set is a CDS, but not a CDStree. Then, by Definition 6, there exists a process $P_{i}$ with $\operatorname{dom}\left(P_{i}\right)=$ true, but neither $P_{i}$ nor its children are the members of the MIS. Then, the guard of GC5 is true at $P_{i}$. This is a contradiction for the assumption that no process is privileged in $\gamma$.

Therefore, the Condition 3 is true in $\gamma$.

Therefore, $\gamma \in \Lambda_{C}$ if no process is privileged.

It is clear that no process is privileged if the configuration is legitimate.

Lemma 2 For any configuration $\gamma_{0}$ and any computation starting from $\gamma_{0}$, eventually no process is privileged.

Proof: First, the root process $P_{r}$ with $L\left(P_{r}\right)=0$ executes $\mathrm{GC} 1$ at most once, and decides the values of $\operatorname{ind}\left(P_{r}\right)=$ true and $\operatorname{dom}\left(P_{r}\right)=$ true. These values never change after that, because they are not changed by other guarded-commands. Therefore, we suppose below that the values of them are correct at $P_{r}$.

By the definition of SS-CDS, at $P_{i} \neq P_{r}$, the value of ind $\left(P_{i}\right)$ is decided by GC2 or GC3, and these guardedcommands do not refer to the values of $\operatorname{dom}\left(P_{j}\right)$ of any processes $P_{j}$. Therefore, the value of $\operatorname{ind}\left(P_{i}\right)$ is decided before the value of $\operatorname{dom}\left(P_{i}\right)$ is decided at each process $P_{i}$.

Suppose that there exists an infinite (non-converging) computation starting from $\gamma_{0}$. Then, there is a process $P_{i}$ such that $L\left(P_{i}\right) \neq 0$ which executes infinitely often.
- Suppose that $P_{i}$ with $L\left(P_{i}\right)=1$ changes the value of $\operatorname{ind}\left(P_{i}\right)$ infinitely often, i.e., $P_{i}$ executes GC2 and GC3 alternately infinitely often. However, because ind $\left(P_{r}\right)=$ true holds at $P_{r}$ and the value never change, $P_{i}$ cannot execute $\mathrm{GC} 2$ by the definition of algorithm. That is, $P_{i}$ executes GC3 at most once. Therefore, $P_{i}$ with $L\left(P_{i}\right)=1$ cannot execute infinitely often.

- Suppose that $P_{i}$ with $L\left(P_{i}\right)=d(d>1)$ changes the value of $\operatorname{ind}\left(P_{i}\right)$ infinitely often, and suppose that $P_{h}$ with $L\left(P_{h}\right)=d-1$ never execute. Then, $P_{j} \in N_{i}$ with $L\left(P_{j}\right)=d$ must change the value of $\operatorname{ind}\left(P_{j}\right)$ infinitely often by the definition of GC2 and GC3. If there exists a process $P_{h} \in N_{i}$ with $L\left(P_{h}\right)=d-1$ and $\operatorname{ind}\left(P_{h}\right)=$ true, then $P_{i}$ cannot execute GC2. Then, $P_{i}$ cannot execute GC3 infinitely often. Therefore, there exist no process $P_{h} \in N_{i}$ with $L\left(P_{h}\right)=d-1$ and $\operatorname{ind}\left(P_{h}\right)=$ true. However, $P_{i}$ can change the value of ind $\left(P_{i}\right)$ from false to true by GC2 only when all its neighbor $P_{j}$ with $L\left(P_{j}\right) \leq d$ hold ind $\left(P_{j}\right)=$ false by the definition of GC2. After $P_{i}$ executes GC2, the guard of GC2 cannot become true at all of its neighbor $P_{j}$ with $L\left(P_{j}\right)=d$. Therefore, $P_{j}$ cannot change the value of ind $\left(P_{j}\right)$ infinitely often. This is a contradiction for the assumption. Therefore, $P_{i}$ and $P_{j}$ cannot execute GC2 and GC3 infinitely often.

There is no process which executes GC2 and GC3 infinitely often. We suppose below that the value of $i n d\left(P_{i}\right)$ of each $P_{i}$ is correct, and never change.

Suppose that $P_{i}$ changes $\operatorname{dom}\left(P_{i}\right)$ infinitely often, i.e., $P_{i}$ executes GC4 and GC5 alternately infinitely often. However, by the definition of GC4 and GC5, each process $P_{i}$ decides the value of $\operatorname{dom}\left(P_{i}\right)$ based only on the values of $\operatorname{ind}\left(P_{i}\right)$ and each $\operatorname{ind}\left(P_{j}\right)$ where $P_{j}$ is a child of $P_{i}$ on $T$. This is a contradiction for the assumption that the value of $\operatorname{ind}\left(P_{i}\right)$ of each process $P_{i}$ never change. The value of $\operatorname{dom}\left(P_{i}\right)$ changes at most once.

Therefore, $P_{i}$ cannot execute infinitely often.

Theorem 3 The algorithm SS-CDS is self-stabilizing with respect to $\Lambda_{C}$.

Proof: Clear from Lemmas 1 and 2.

Theorem 4 Time complexity of SS-CDS is $O\left(n^{2}\right)$ steps.

Because of limitation of space, proof of this theorem is omitted.

Theorem 5 SS-CDS is a self-stabilizing approximation algorithm for the minimum CDS problem in unit disk graphs. The size of the solution by SS-CDS is at most $8\left|D_{\text {opt }}\right|+1$, where $D_{\text {opt }}$ is a minimum CDS. 
Proof: From Theorem 3, the set $\left\{P_{i} \mid \operatorname{dom}\left(P_{i}\right)=\right.$ true $\}$ is a CDS-tree. From Theorem 2, the size of the solution by SS-CDS is at most $8\left|D_{\text {opt }}\right|+1$.

\section{Conclusion}

In this paper, we proposed a self-stabilizing distributed approximation algorithm for the minimum CDS in unit disk graphs. As an application of the proposed algorithm, a minimum CDS is a virtual backbone or routing in mobile ad hoc networks. Since our algorithm is self-stabilizing, it is strongly desirable in mobile ad hoc networks.

The size of the solution by our algorithm is at most $8\left|D_{\text {opt }}\right|+1$, where $D_{\text {opt }}$ is a minimum CDS. The time complexity is $O\left(n^{2}\right)$ steps. Development of a self-stabilizing approximation algorithm with better approximation ratio is left for future work.

\section{Acknowledgment}

This work is supported in part by JSPS: Grant-in-Aid for Scientific Research ((B)17300020) and Ookawa Foundation Research Grant.

\section{References}

[1] V. Bharghavan and B.Das. Routing in ad hoc networks using minimum connected dominating sets. In Proceedings of International Conference on Communications'97, pages 376-380, 1997.

[2] J. Blum, M. Ding, A. Thaeler, and X. Cheng. Connected Dominating Set in Sensor Networks and MANETs, pages 329-369. Kluwer Academic Publishers, 2004.

[3] X. Cheng and D.-Z. Du. Virtual backbone-based routing in multihop ad hoc wireless networks. Technical report, University of Minnesota, 2002.

[4] B. N. Clark, C. J. Colbourn, and D. S. Johnson. Unit disk graphs. Discrete Mathematics, 86:165-177, 1990.

[5] E. W. Dijkstra. Self-stabilizing systems in spite of distributed control. Communications of the ACM, 17(11):643-644, 1974.

[6] S. Dolev. Self-Stabilization. The MIT Press, 2000.

[7] B. Gao, Y. Yang, and H. Ma. A new distributed approximation algorithm for constructing minimum connected dominating set in wireless ad hoc networks. International Journal of Communication System, 18:743-762, 2005.
[8] S. Guha and S. Khuller. Approximation Algorithms for Connected Dominating Sets. Algorithmica, 20:347-387, 1998.

[9] S. M. Hedetniemi, S. T. Hedetniemi, D. P. Jacobs, and P. K. Srimani. Self-stabilizing algorithms for minimal dominating sets and maximal independent sets. Computers and Mathematics with Applications, 46(56):805-811, 2003.

[10] S. T. Huang and N.-S. Chen. A self-stabilizing algorithm for constructing breadth first trees. Information Processing Letters, 41(2):109-117, 1992.

[11] A. Jain and A. Gupta. A distributed self-stabilizing algorithm for finding a connected dominating set in a graph. In Proceedings of the 6th International Conference on Parallel and Distributed Computing, Applications and Technologies (PDCAT'05), pages 615-619. IEEE Press, 2005.

[12] M. V. Marathe, H. Breu, H. B. Hunt III, S. S. Ravi, and D. J. Rosenkrantz. Simple heuristics for unit disk graphs. Networks, 25:59-68, 1995.

[13] E. Sampathkumar and H. B. Walikar. The connected domination number of a graph. Journal of Mathematical Physical Sciences, 13:607-613, 1979.

[14] S. K. Shukla, D. J. Rosenkrantz, and S.S.Ravi. Observations on self-stabilizing graph algorithms for anonymous networks. In Proceedings of the 2nd Workshop on Self-Stabilizing Systems (WSS'95), pages 7.17.15. Chicago Journal of Theoretical Computer Science, 1995.

[15] S. Sur and P. K. Srimani. A self-stabilizing distributed algorithm to construct BFS spanning trees of a symmetric graph. Parallel Processing Letters, 2:171-179, 1992.

[16] P.-J. Wan, K. M. Alzoubi, and O. Frieder. Distributed construction of connected dominating set in wireless ad hoc networks. Mobile Networks and Applications, 9:141-149, 2004.

[17] J. Wu and H.L. Li. On calculating connected dominating set for efficient routing in ad hoc wireless networks. In Proceedings of the 3rd ACM International Workshop on Discrete Algorithms and Methods for Mobile Computing and Communications, pages 7-14, 1999.

[18] J. Wu and W. Lou. Forward-node-set-based broadcast in clustered mobile ad hoc networks. Wireless Networks and Mobile Computing, 3(2):155-173, 2003. 